\title{
The Research of Vibration Signal De-noising Method Based on LMD and Wavelet De-noise
}

\author{
GUO gangxiang ${ }^{1, a}$, DING Yuanming ${ }^{1, b}$, CHEN jinwei ${ }^{1, c}$ \\ ${ }^{1}$ Zhejiang Province Institute of Metrology, Hangzhou Zhejiang 310027, China \\ a guanagu@163.com, byuanming.ding@gmail.com, ${ }^{\mathrm{c}}$ chenjinwei_1987@126.com
}

Keywords: signal de-noising, LMD, wavelet

Abstract. This paper puts forward a vibration signal de-noising method based on LMD and Wavelet De-noise. The method de-noises the PFs separated from LMD by wavelet De-noise, and reconstitute the de-noised signal. It's effective to de-noising diesel engine vibration signal and simulation signal. Compared with wavelet noise reduction method, the method is better at de-noising amplitude \& frequency modulated signal de-noising.

\section{Introduction}

Signal noise reduction is an important step in fault diagnosis. This step makes signal more accurately by getting rid of the noise. This paper takes two diesel engine working condition which is normal condition and fuel supply advance angle fault as testing signal, and choosing a simulate amplitude \& frequency modulated signal as a comparing signal. Figure 1 is one period signal of diesel engine in normal-working condition[1]. The simulate amplitude \& frequency modulated signal is:

$$
x=2+\cos (3 t) \times \cos \left(2 t+2 t^{2}\right)+0.2 \text { randn } \quad t \in[0,3] .
$$

$x=2+\cos (3 t) \times \cos \left(2 t+2 t^{2}\right)$ is the true part of this simulate amplitude \& frequency modulated signal. In figure 2, picture (a) is the noisy overlapped signal, and picture b is the true part of this simulate signal.

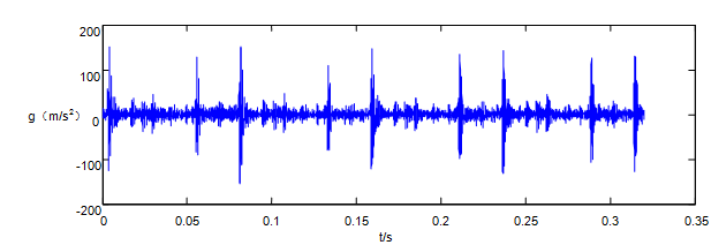

Fig. 1 One period signal of diesel engine in normal-working condition

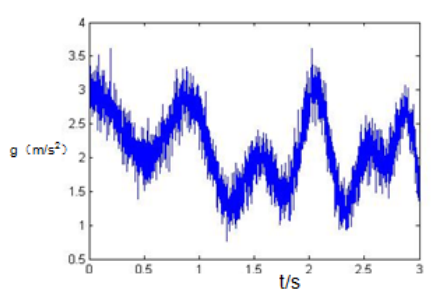

(a). The noisy overlapped signal

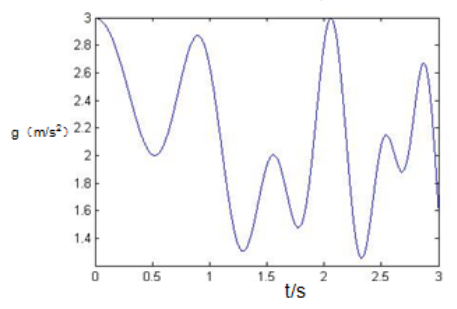

(b). The true part of this simulate signal

Fig. 2 The simulate signal

\section{De-noising signal base on LMD and wavelet de-noise}

2.1 De-noising the simulate signal based on LMD and Wavelet De-noise.8 PFs separated from the simulate signal by LMD. Because of the noise, PFs have some major peaks. This paper uses wavelet de-noise to remove noise[2]. According to different frequency PFs, different threshold values are applied to PF's wavelet de-noise[3]. Figure 3 is the comparison of the PF4 wavelet 
de-noising. We can find that a lot of noise is de-noised, and get the de-noised signal. By Accumulating all de-noised PFs, simulate signal de-noising is completed. Figure 4 is the comparison of simulate signal de-noising.

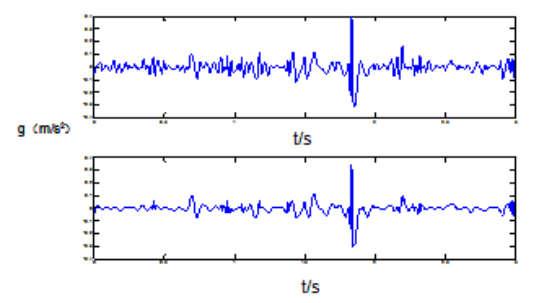

Fig.3 The comparison of PF4 and PF4 LMD- Wavelet de-noising signal

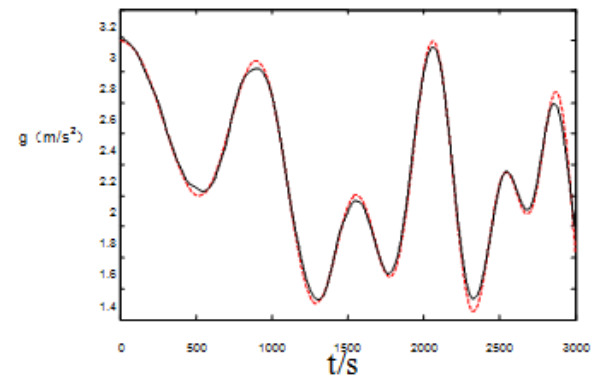

Fig 4 The comparison of the original signal and LMD- Wavelet de-noising signal

2.2 De-noising diesel engine vibration signal based on LMD and Wavelet De-noise. Firstly , diesel engine vibration signal of two diesel engine working condition is separated to some PFs, then this PFs are wavelet de-noised with different threshold value, finally, we can get the de-noising diesel engine vibration signal by reconstituting the de-noised PFs. Figure5 is the comparison of diesel engine original signal and LMD- wavelet de-noising signal.
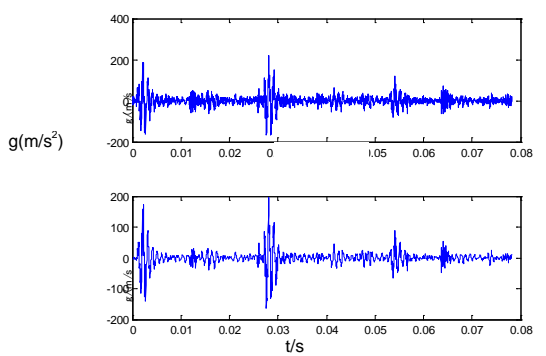

(a) normal condition
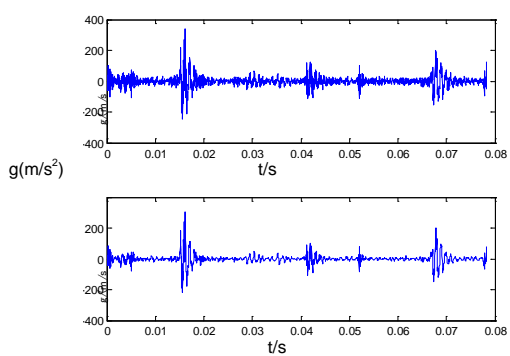

(b) fuel supply advance angle fault

Fig. 5 The comparison of diesel engine signal and LMD- wavelet de-noising signal

\section{Wavelet Threshold De-noising}

3.1 Wavelet Threshold De-noising for Simulate Signal Function wdencmpthreshold de-noises the simulate signal, which function is used in one-dimensional or two-dimensional signal de-noised. The format of the function follows[4]:

$$
X=\text { wdencmp ('gbl', } X \text {, 'wname', } N, T H R, \text { SORH.KEEPAPP) }
$$

Where 'vname' is wavelet function of this paper, ' $g b l$ ' (the abbreviation of global .) express that one wavelet decomposition level to one threshold value, $N$ expresses the wavelet decomposition level, THR is threshold value return.

We can get the comparison of the original signal and Wavelet de-noising signal by the above algorithm. The dashed part is the real part of the original signal, and solid part is noise reduction of the original signal in the figure 6. 


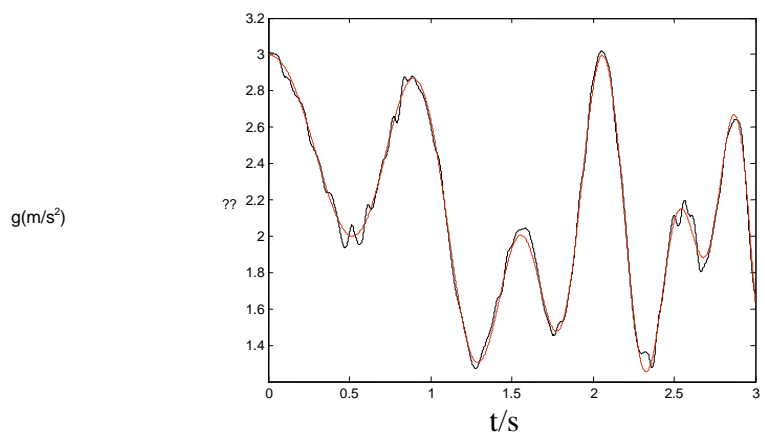

Fig. 6 The comparison of the original signal and Wavelet de-noising signal

3.2 De-noising diesel engine vibration signal based on Wavelet Threshold De-noisingDiesel engine vibration signal of two diesel engine working condition is de-noised by wavelet threshold de-noising. De-noising is effective. Figure7 is the comparison of diesel engine original signal and wavelet de-noising signal.

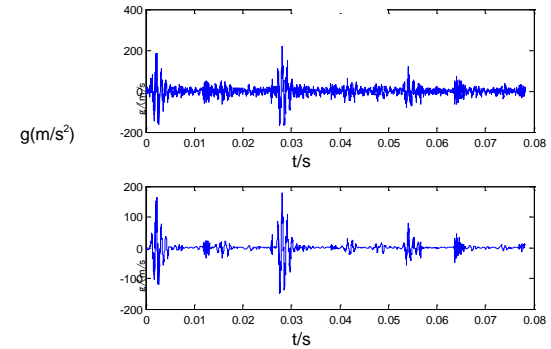

(a) normal condition
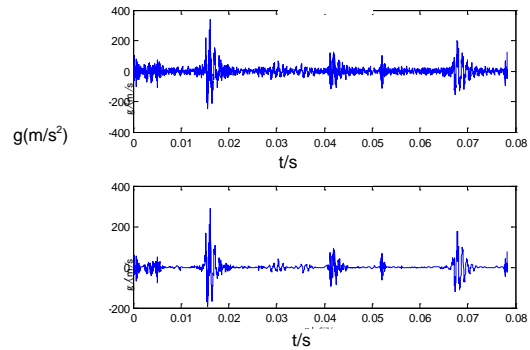

(b) fuel supply advance angle fault

Fig.7 The comparison of diesel engine original signal and wavelet de-noising signal

\section{Comparison of two de-noise method's de-noising effect}

Observing figure4 and figure6, we can find that the de-noised signal from Wavelet de-noising or LMD- Wavelet de-noising is close to real part of the origin signal. The de-noising effect is obvious. This paper takes the variance of the original signal and de-noising signal as the evaluation index. The function as follows:

$$
S=\sum_{i=1}^{k}[y(i)-x(i)]^{2}
$$

Where $S$ is the variance of the original signal and de-noising signal, $y(i)$ is de-noising signal, $x(i)$ is original signal.

This paper calculates variance of two de-noise method by matlab, the result as follows:

Table 1 The variance of two de-noise method

\begin{tabular}{cc}
\hline De-noising method & Variance $(\mathrm{S})$ \\
\hline Based on Wavelet de-noising & 4.1652 \\
Based on LMD- Wavelet de-noising & 2.9950
\end{tabular}

From sheet 1, we can get that the variance of the method based on LMD- Wavelet de-noising is 2.9950, smaller than the variance of the method based on wavelet de-noising. The result is in agreement that we compare the figure4 and figure6.

Different signal de-noised by different de-noising method, there is different effect for the same de-noising method applying to the other signal. This paper takes Mean Square Error and Mean Square Error to evaluate the result of diesel engine signal de-noising: the smaller MSE and the bigger SNR, the better de-noising effect. The definition of MSE and SNR follows[5]: 


$$
\begin{gathered}
M S E=\frac{1}{N} \sum_{k=1}^{N}(x(k)-\hat{x}(k))^{2} \\
S N R=10 \log \left[\frac{\sum_{k=1}^{N} x^{2}(k)}{\sum_{k=1}^{N}(x(k)-\hat{x}(k))^{2}}\right]
\end{gathered}
$$

Where $x(i)$ is the after de-noised signal, $\hat{x}(k)$ is the origin signal, $\mathrm{N}$ is the number of the origin signal.

Table 2 Mean Square Error and Mean Square Error of two de-noise method

\begin{tabular}{cccc}
\hline Fault type & Estimate & $\begin{array}{c}\text { Wavelet } \\
\text { De-noising }\end{array}$ & $\begin{array}{c}\text { LMD- Wavelet } \\
\text { De-noising }\end{array}$ \\
\hline Normal Condition & SNR & 9.0054 & 16.0180 \\
& MSE & 150.37 & 116.47 \\
Fuel Supply & SNR & 11.1640 & 15.654 \\
Advance Angle & MSE & 187.57 & 152.12 \\
Fault & & & \\
\hline
\end{tabular}

It is obvious that the vibration signal de-noising method based on LMD and Wavelet De-noising gets higher SNR and smaller MSE than the method of Wavelet De-noising in de-noising diesel engine signal of two conditions. The method raised in this paper represents the diesel engine vibration signal accurately.

\section{Conclusion}

The vibration signal de-noising method of this paper based on LMD and Wavelet De-noise is applied to amplitude \& frequency modulated signal. Thought fractional frequency and restructure, it is effective for de-noising diesel engine vibration signal and simulation signal. Compared with wavelet noise reduction method, this method gets higher SNR and smaller MSE, and keeps the inherent characteristic of the origin signal for future signal processing.

\section{Acknowledgements}

This paper is supported by a project of Zhejiang province science and technology hall which is the research of underwater coded structured light stereo vision odometry.

\section{References}

[1] Alan V.Oppenheim,A.S.W..Signals and system 2nd ed[M].London:Prentice-Hall. 1997

[2] Jonathan S.Smith.The loeal mean deeomposition and its aPPlieation to EEG PercePtion data[J].Journal of the Royal Society Inteface,2005,2(5):444-450

[3] Wu J D,Kuo J M.An automotive generator fault diagnosis system using diserete wavelet transform and artificial neural network[J].ExPert Systems with APPlieations,2009,36(6):9776-9783

[4] Wu J D,Kuo J M.An automotive generator fault diagnosis system using diserete wavelet transform and artificial neural network[J].ExPert Systems with APPlieations,2009,36(6):9776-9783

[5] Zhen-wen Qian,Li Chen,Ying-hong. The signal de-noising method based on singular value decomposition[J]. Vibration, measurement \& diagnosis, 2011,31(4):459-463 